\title{
Universal Design in the Metrobuss System of Trondheim, Norway - Challenges and Solutions
}

\author{
Jacob DEICHMANN ${ }^{1}$ \\ Rambøll Danmark AS
}

\begin{abstract}
The presentation describes challenges and possible solutions for achieving truly accessible high-class urban public transportation based on a case from Trondheim, where a new high-class bus system was implemented. The implemented solution did not reflect the wheelchair user's needs- despite clearly stated ambitions for accessibility.
\end{abstract}

Ramboll conducted a study comprising a screening of the international market for relevant solutions, combined with interviews with representatives of Public transport authorities. The results were presented to the local user's representatives, and some solutions tested on location. Based on this process, recommendations for short-, medium- and long-term solutions were made.

The project highlights the need for involvement of sufficient professional knowledge of universal design in the planning phase as well as in the implementation phase.

Keywords. Accessibility, public transport

\section{Introduction, definition and motivation}

\subsection{Introduction}

The Metrobuss system opened in Trondheim, Norway, on August $3^{\text {rd }}, 2019$.

The system consists of 3 bus lines in a quasi BRT-standard (Bus Rapid Transit). Focus in the planning and design were on the rolling stock and the design of the stations in the system. From the outset of the planning process, the ambitions for Universal Design and Accessibility were high, reflecting the generally high level of inclusion in Norway.

At a late stage in the planning process, with construction of the stations and delivery of the buses well underway, it was discovered that the stations and the buses had been built to different accessibility standards, making unaided travel with the buses impossible for wheel chair users and other passengers with physical impairments.

${ }^{1}$ Jacob Deichmann, Rambøll Denmark AS, Hannemanns Allé 53, DK-2300 København S, Denmark, jpd@ramboll.dk 
The main topic of this paper is the accessibility for wheelchair users in the buses. This is however used as a starting point for a discussion of the general challenges of achieving a truly universally designed public transport system.

\subsection{Trondheim}

Trondheim is a city in Central Norway with app. 180,000 inhabitants, making it Norway's third largest city.

Apart from a single and somewhat outdated tram line, until August 2019 Trondheim did not have an efficient system of high-class public transport within the urban area.

\subsection{Definition of BRT}

Bus rapid transit (BRT) is a bus-based public transport system designed to improve capacity and reliability relative to a conventional bus system. Typically, a BRT system includes roadways that are dedicated to buses, and gives priority to buses at intersections where buses may interact with other traffic; alongside design features to reduce delays caused by passengers boarding or leaving buses, or purchasing fares. BRT aims to combine the capacity and speed of a metro with the flexibility, lower cost and simplicity of a bus system [1].

A BRT system will usually consist of a combination of the below listed components:

1. An alignment that prioritizes the bus, usually with segregated bus lanes on as much of the alignment as possible.

2. Specially designed stations in a higher standard than a usual bus stop

3. Specially designed rolling stock, often longer buses with extra capacity.

4. A timetable with a high frequency to reduce waiting time at stops and transfers.

The "Metrobuss" system in Trondheim features components nos 2-4. The 3 lines have a base frequency of 10 minutes but run more often in rush-hours.

- 130 stations (bus stops) have been built or extended.

- 58 new 24 m-long buses were acquired to serve the 3 Metrobuss lines.

The construction and implementation of the Metrobuss system were financed partly by a congestion charge for cars entering Trondheim and is part of a larger package of infrastructure projects aimed at encouraging the population of Trondheim to make more sustainable choices of transportation mode.

\subsection{Definition of Universal design and interpretation in relation to Public Transport}

The United Nations' Convention on the Rights of Persons with Disabilities, article 2, has the following definition of Universal design:

"Universal design" means the design of products, environments, programmes and services to be usable by all people, to the greatest extent possible, without the need for adaptation or specialized design. "Universal design" shall not exclude assistive devices for particular groups of persons with disabilities where this is needed [4]. 
In terms of public transport this can be interpreted as a system where all groups of passengers can use the same system unaided. The concept must cover access to stations, design of the stations, accessibility between station and rolling stock (e.g. buses, trams or trains), internal design of the rolling stock, as well as timetable and operational information given on websites and information given at the station and in the rolling stock (information on destination, next stop, delays etc).

Public transport plays a central role in the mobility of people with disabilities and their possibilities of leading a normal, full life with jobs, studies etc., as many in this group cannot obtain a driver's license because of their impairments.

\section{Construction and procurement - observed challenges}

From the outset, the Metrobuss project had high ambitions on accessibility, in line with the general Norwegian approach.

The stations were built according to Norwegian State guidelines for accessible road design [2]. A central feature of bus stop design here is a kerb that is $18 \mathrm{~cm}$ high. The kerb has a slightly receding profile, with a curve at the bottom, designed to encourage the bus driver to drive as close to the kerb as possible (Figure 1, left)
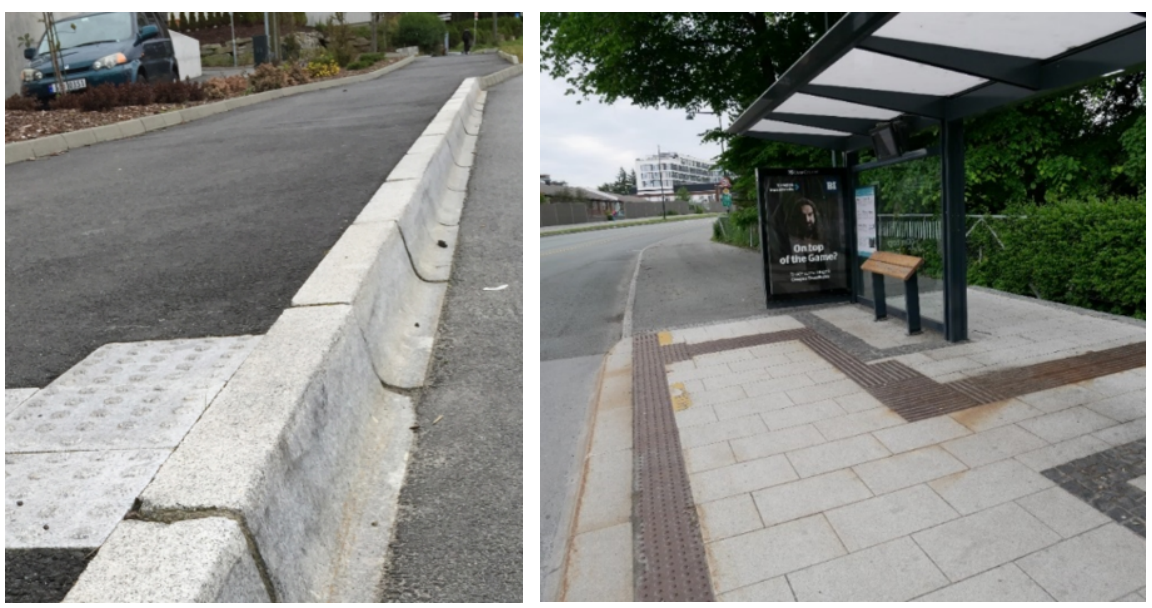

Figure 1. Left: Detail of the $18 \mathrm{~cm}$ kerb (photo: Manufacturer).

Right: Tactile surfaces at a Metrobuss station (photo: the author)

The platforms are also fitted with a system of tactile guiding surfaces, primarily to aid blind and visually impaired passengers to find the front door of the bus. (Figure 1, right).

The buses were procured according to the EU Bus directive 2001/85 [4].

According to the section on "requirements for technical devices facilitating access for passengers with reduced mobility":

The height of the first step from the ground of at least one service door shall not exceed $250 \mathrm{~mm}$ for vehicles of Class I and A and $320 \mathrm{~mm}$ for vehicles of Class II, III and 
B. As an alternative for vehicles of Class I and A, the first step from the ground shall not exceed $270 \mathrm{~mm}$ in two door openings, one entrance and one exit.

A kneeling system and/or retractable step may be engaged.

The chosen bus type was the Equicity from the manufacturer VanHool of Belgium.

The bus can reach a floor level at the doors of 25-27 cm by "kneeling" (meaning that one side of the bus is lowered at bus stops using a hydraulic mechanism).

The BRT standard [1] allows for a maximum gap of $5 \mathrm{~cm}$ between platform and bus floor (which is even a large gap for some wheelchair and walker users). A kerb height of $18 \mathrm{~cm}$ and a bus floor level of 25-27 cm results in a vertical gap of 7-9 cms. To this can be added the horizontal gap between bus and kerb edge. This gap depends very much on the skill of the driver and the detail of the kerb design.

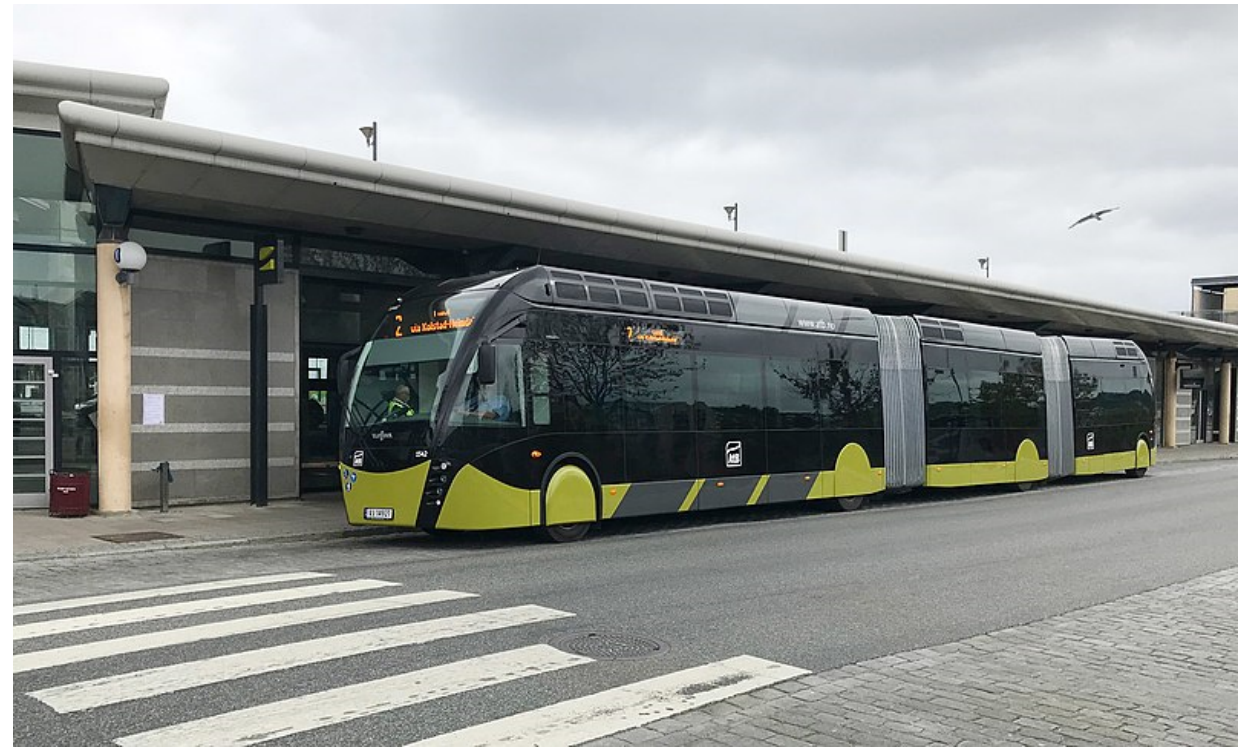

Figure 2. A VanHool Equicity Bus (photo Wikipedia)

At some time during the construction and procurement process this discrepancy was discovered, and the users' organizations complained to the political system and in the media.

$\mathrm{AtB}$, the transport authority replied to the criticism by stating that both stations and buses are built to the best available standards of accessibility, and that all buses would be fitted with manual flip ramps.

However, manual flip ramps can only be activated either by the bus driver, who must leave his seat, by a person accompanying the wheelchair user, or by a random other passenger present in the bus.

None of these solutions are acceptable: The driver leaving his seat will extend the bus' stay at the bus stop and delay all passengers in the bus. This is at odds with the overall purpose of the Metrobuss system: Creating a system that can compete with individual car travel. An accompanying person is a requirement which contrasts with the principle that the user should be able to travel on his/her own. Finally, depending on 
random passengers will remove the dignity of travel and make the disabled passenger depending on chance in an unacceptable way.

In AtB's statement, it was revealed that motorized ramps had been considered, but not chosen, due to anticipated maintenance issues.

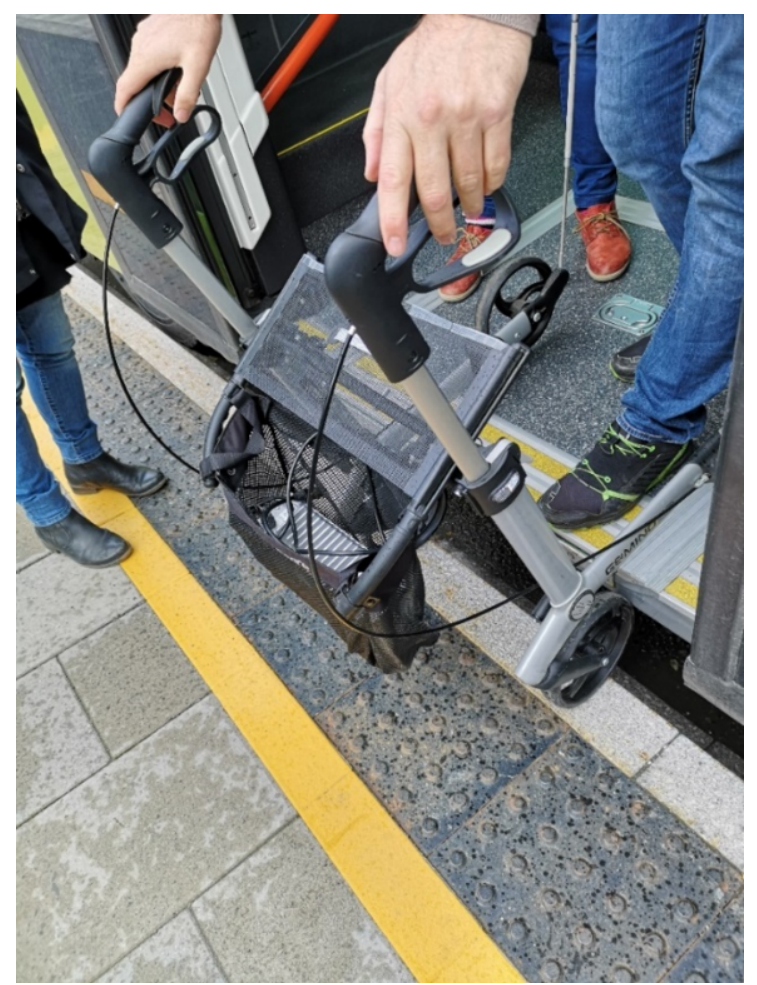

Figure 3. Vertical and horizontal gap shown by a rollator (walker) user (photo: Rambøll)

At this point it was decided to involve an external consultant to evaluate the situation and suggest a way forward. After a tender process Rambøll was chosen to do the task with a Norwegian project team, and the author (Dane) attached as expert.

\section{Contents of the research project}

\subsection{Overview}

The project agreed between Trondheim Kommune (Municipality of Trondheim), AtB (the transit authority) and Rambøll consisted of these activities:

1. Desk top research and contacts in the network to discover status of possible solutions used in other countries.

2. User trials using one of the already delivered buses.

3. Defining a range of realistic solutions, and ranking them after relevance.

4. Suggesting a reduced number of solutions to be tested full-scale.

In general, a high level of user involvement was deemed essential. 


\subsection{Desk top research}

\subsubsection{Methodology}

The desk top research consisted of internet searches, contacts in the professional network and interviews with manufacturers at a public transport conference in Stockholm in June 2019.

\subsubsection{Results, desk top research}

In the Nordic Countries and Germany, almost no buses are fitted with motorized ramps. Most buses are fitted with manual ramps, but the use of these requires either that that the driver leaves the steering wheel and flips out the ramp, or that this is done by a person accompanying the wheelchair user or by a random passenger entering or present in the bus. None of these solutions are acceptable from the universal design point of view.

The reasons for not using motorized ramps were reported as anticipated problems with snow, dead leaves, high cost and general maintenance.

Skånetrafiken, the regional transit authority of South Sweden, states that they have fitted manual flip ramps, and the users seem to be happy with this. Motorized ramps were not chosen due to anticipated maintenance issues (gravel). [5].

As opposed to this, at least many city buses in London, UK, and Paris, France, are fitted with motorized ramps, as this is required by national legislation.

Motorized ramps are also used in Vancouver, Canada, a city that has weather conditions similar to Trondheim's.

In addition to the desk top research, the author participated in the UITP conference on Public Transport in Stockholm and had the opportunity to discuss the problem with some manufacturers of bus equipment. From these discussions it was evident that usable solutions are certainly available on the regular market for bus equipment.

\subsection{User trials}

\subsubsection{Methodology}

Two full-scale user trials were conducted.

One of the buses that had already been delivered was moved to one of the stations and a number of tests were carried out, including

- How close to the kerb the bus could be located during the stop maneuver.

- How much the bus could kneel.

- Entering and exiting the bus with or without the manual ramp employed.

Participants were representatives of The Norwegian Association of Disabled, The Municipality of Trondheim, The Region of Trøndelag, The Norwegian State Road Authority and the Transit Authority AtB.

Among the user representatives were wheelchair users (motorized and manual), walker users and blind and visually impaired people. 


\subsubsection{Results, user trials}

The bus can actually kneel to a floor height of 23-24 cm, which is lower than required in the specifications and only $5-6 \mathrm{~cm}$ above the kerb. But in this case the inclination of the floor inside the bus is up to $8 \%$, resulting in other challenges.

The manual ramp is too narrow and feels unsafe. When open, the ramp leaves a recess in the floor inside the bus, which poses a problem for some wheelchair users.

Due to the design of the wheel casings the bus cannot get as close to the kerb as expected or needed, meaning that the horizontal gap remains a problem; even if the bus floor can come closer to the kerb height.

There is generally too little colour contrast in the interior bus design, e.g. wheelchair symbols and handles as opposed to seats.

In addition to the user trials, a workshop with user representatives and technical experts was carried out. The workshop was used to structure and qualify the results of the user trials.

\subsection{Suggesting a number of solutions to be assessed for future testing}

\subsubsection{Methodology}

Based on the inputs from the desk top research and the user tests, several solutions were chosen to be assessed:

1. Manual ramp (as existing in the buses), baseline

2. Motorized ramp (flip)

3. Motorized ramp (telescope)

4. Gap filler for vehicle

5. Gap filler for platform

6. Mechanical guiding system

7. Optical guiding system

8. Bus pad

9. Raised kerb level

As well as 3 combined solutions:

10. Bus pad + guiding system

11. Raised kerb level + guiding system

12. Gap filler for vehicle + guiding system

Each solution was scored with points from 1 to 5 ( 5 being best) for each of these parameters:

- Solving the horizontal gap

- Solving the vertical gap

- Functionality and universal design

- Installation, maintenance and aesthetics

- Commercial availability

- Cost 
As an additional solution for short-term a training program for the bus drivers was suggested. The focus should be on identifying passengers with special needs, and on getting as close to the kerb as possible.

And the chosen solutions were ranged in short-term (within 1 year), medium-term (2 -10 years) and long term ( $>10$ years, corresponding to the next tender of the buses).

\subsection{Grading of the solutions}

\begin{tabular}{|c|c|c|c|c|c|c|c|c|c|c|}
\hline No. & Solution & 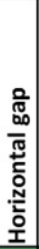 & 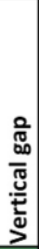 & 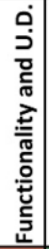 & 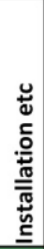 & 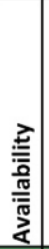 & 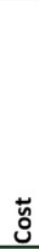 & Time frame & 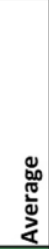 & 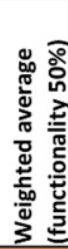 \\
\hline 1 & Manual ramp (baseline) & 5 & 5 & 1 & 5 & 5 & 5 & Existing/baseline & 4,3 & 3,0 \\
\hline 2 & Motorized ramp (flip) & 5 & 5 & 3 & 3 & 1 & 2 & Medium term & 3,2 & 3,1 \\
\hline 3 & Motorized ramp (telescope) & 5 & 5 & 4 & 3 & 5 & 2 & Medium term & 4,0 & 4,0 \\
\hline 4 & Gap filler, vehicle & 4 & 5 & 4 & 3 & 0 & 1 & Long-term & 2,8 & 3,3 \\
\hline 5 & Gap filler, platform & 3 & 0 & 3 & 1 & 5 & 4 & Short-term & 2,7 & 2,8 \\
\hline 6 & Mechanical guiding system & 5 & 0 & 2,5 & 4 & 3 & 3 & Short-term & 2,9 & 2,8 \\
\hline 7 & Optical guiding system & 5 & 0 & 2,5 & 5 & 5 & 2 & Medium/long & 3,3 & 3,0 \\
\hline 8 & Bus pad & 0 & 5 & 3 & 2 & 5 & 4 & Short/medium & 3,2 & 3,1 \\
\hline 9 & Raised kerb level & 0 & 5 & 5 & 1 & 4 & 1 & Long-term & 2,7 & 3,6 \\
\hline 10 & Bus pad + guiding system & 5 & 5 & 3 & 2,5 & 5 & 3 & Short/medium & 3,9 & 3,6 \\
\hline 11 & Raised kerb + guiding system & 5 & 5 & 5 & 3 & 5 & 1 & Long-term & 4,0 & 4,4 \\
\hline 12 & Gap filler (veh) + guiding system & 5 & 5 & 5 & 3 & 2 & 1 & Long-term & 3,5 & 4,1 \\
\hline
\end{tabular}

Figure 4. Scoring table of the solutions investigated.

The following section contains short descriptions of each solution, as well as the assessment by the project team.

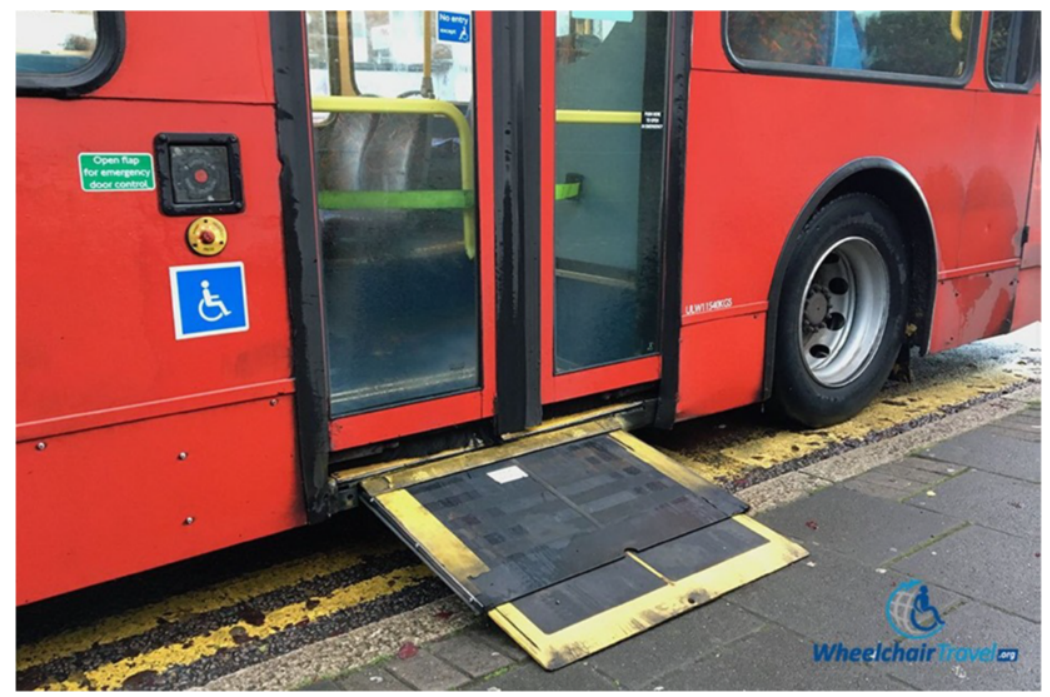

Figure 5. Motorized ramp, telescope system (solution no. 3) 


\subsubsection{Descriptions and assessment}

The two different numbers under scoring represent unweighted average / average with functionality and accessibility weighing $50 \%$, respectively.

\begin{tabular}{|c|c|c|c|}
\hline No. & Solution & Description & Scoring \\
\hline 1 & $\begin{array}{l}\text { Manual ramp (as } \\
\text { existing in the buses) }\end{array}$ & $\begin{array}{l}\text { The manual ramp is already fitted in all } \\
\text { buses and serves as kind of baseline to the } \\
\text { other solutions. As described previously, it } \\
\text { can only be activated either by the driver, } \\
\text { who must leave his seat, by a person } \\
\text { accompanying the wheelchair user or by a } \\
\text { random other passenger present in the bus. }\end{array}$ & Score $4.3 / 3.0$ \\
\hline 2 & Motorized ramp (flip) & $\begin{array}{l}\text { In this solution the ramp flips out of the } \\
\text { bus in the same way as the manual ramp } \\
\text { but aided by a motor. The use requires that } \\
\text { space is freed in the bus as well as on the } \\
\text { platform, and there may be risk to waiting } \\
\text { passengers on the platform. The motorized } \\
\text { ramp was not found to be commercially } \\
\text { available. }\end{array}$ & Score 3.2./ 3.1 \\
\hline 3 & $\begin{array}{l}\text { Motorized Ramp } \\
\text { (Telescope) }\end{array}$ & $\begin{array}{l}\text { In this solution the ramp is mounted under } \\
\text { the bus, protrudes horizontally and is then } \\
\text { lowered to the platform level. In a video } \\
\text { from the solution in London it appears that } \\
\text { all other passengers must enter and leave } \\
\text { the bus and the door be closed and then } \\
\text { reopened before the ramp can be activated. } \\
\text { If this is the case, there will be delays } \\
\text { connected to the use. But according to } \\
\text { information received from Fynbus [6] in } \\
\text { Denmark, no such requirements exist } \\
\text { there. } \\
\text { The solution does not affect passengers in } \\
\text { the bus, but the space where the ramp } \\
\text { protrudes must be marked on the platform } \\
\text { and the movement of the ramp must be } \\
\text { signaled by sound and light signals. } \\
\text { It is complicated to fit the ramp on an } \\
\text { existing bus, but ramps can be easily fitted } \\
\text { on new buses. }\end{array}$ & Score $4.0 / 4.0$ \\
\hline 4 & Gap filler for vehicle & $\begin{array}{l}\text { The gap filler is a kind of short ramp that } \\
\text { protrudes horizontally from the vehicle-- } \\
\text { or flips out from the bus - and connects it } \\
\text { to the platform, which will generally have } \\
\text { to be the same height as the bus floor. } \\
\text { Gap fillers are not common on bus } \\
\text { systems but are seen internationally on } \\
\text { light rail and metro lines, where the } \\
\text { distance between vehicle and platform is } \\
\text { fixed due to the rails. In these solutions the } \\
\text { gap filler is usually activated at all stops. }\end{array}$ & Score $2.8 / 3.3$ \\
\hline
\end{tabular}




\begin{tabular}{|c|c|c|c|}
\hline 5 & Gap filler for platform & $\begin{array}{l}\text { The gap filler can also be mounted on the } \\
\text { platform and be permanent (e.g. made of } \\
\text { rubber or similar material) or mechanical. } \\
\text { A gap filler system is known to exist on at } \\
\text { least one BRT system in China. }\end{array}$ & Score $2.7 / 2.8$ \\
\hline 6 & $\begin{array}{l}\text { Mechanical guiding } \\
\text { system }\end{array}$ & \multirow{2}{*}{$\begin{array}{l}\text { Guiding systems can help the bus driver } \\
\text { getting as close to the kerb as possible, } \\
\text { closer than is possible by pure manual } \\
\text { control of the bus. } \\
\text { Two systems exist: In the mechanical } \\
\text { system the bus is guided by small wheels } \\
\text { mounted horizontally on the side of the } \\
\text { bus. } \\
\text { In the optical system, the bus is guided by } \\
\text { a white street marking combined with a } \\
\text { system of video detection mounted on the } \\
\text { front and bottom of the bus }\end{array}$} & Score $2.9 / 2.8$ \\
\hline 7 & Optical guiding system & & Score $3.3 / 3.0$ \\
\hline 8 & Bus pad & $\begin{array}{l}\text { The bus pad is a local raise of the platform } \\
\text { level, located to correspond with one of } \\
\text { the bus doors. The bus pad consists of } \\
\text { element of recycled hard plastic and is } \\
\text { mounted on top of the existing paving. } \\
\text { Use of the bus pad would give challenges } \\
\text { to the use of the tactile guiding systems, } \\
\text { especially if located at the front door of the } \\
\text { bus. }\end{array}$ & Score $3.2 / 3.1$ \\
\hline 9 & Raised kerb level & $\begin{array}{l}\text { This solution involves raising the entire } \\
\text { kerb to a height of e.g. } 23 \mathrm{~cm} \text {, which will } \\
\text { also necessitate a raising of the entire } \\
\text { platform area behind the kerb. }\end{array}$ & Score $2.7 / 3.6$ \\
\hline 10 & $\begin{array}{l}\text { Bus pad + guiding } \\
\text { system }\end{array}$ & & Score $3.9 / 3.6$ \\
\hline 11 & $\begin{array}{l}\text { Raised kerb level + } \\
\text { guiding system }\end{array}$ & & Score $4.0 / 4.4$ \\
\hline 12 & $\begin{array}{l}\text { Gap filler for vehicle }+ \\
\text { guiding system }\end{array}$ & & Score $3.5 / 4.1$ \\
\hline 13 & Driver training & $\begin{array}{l}\text { The suggested training consists of } \\
\text { information on the different user needs as } \\
\text { well in training in parking the bus closer to } \\
\text { the kerb. Among the suggested measures } \\
\text { are the construction of a test area with a } \\
\text { mock-up of a bus stop. }\end{array}$ & Not graded \\
\hline
\end{tabular}




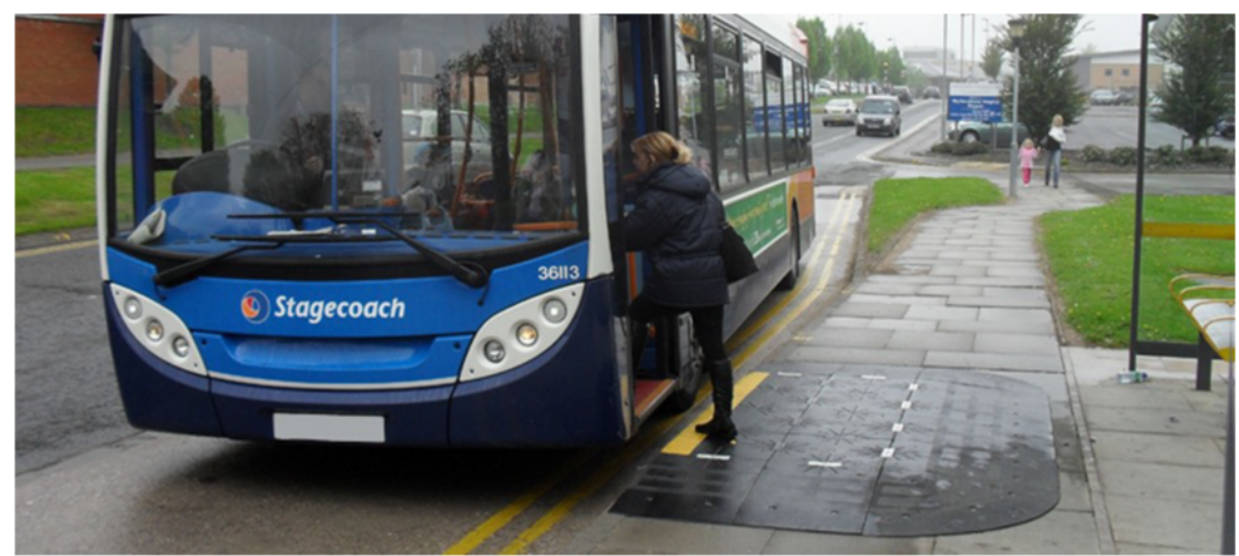

Figure 6. Bus Pad (solution no. 8)

\section{Project proposal and status}

Based on the scoring of the solutions the following proposal was made by the consultants and accepted by the client.

\subsection{Solutions chosen for testing}

\subsubsection{Short term solutions (within weeks/months)}

- Training of drivers, improved routines and follow-up on contract requirements.

\subsubsection{Medium-term: Solutions to be tested in a timeframe of 1-2 years}

The focus for these solutions is to enable step-free entering.

- Motorized ramp, telescope system to be fitted on one bus.

- Bus pad to be mounted on one station and tested

- $\quad$ Guiding system to be tested

4.1.3. Long-term: To be implemented in the next tender of buses

- Gap-filler

- $\quad$ Raised platforms

\subsection{Project status as of March 2021}

The program for bus driver training was carried out in the Autumn of 2019. The effects of the training are not known to the author.

In November 2019, one of the Metrobus buses burnt in the depot because of an error in the floor heating system. It was decided that the replacement should be fitted with a motorized ramp. The new bus has been in regular use since delivery, the results are promising, and an evaluation report will be prepared in 2021 after one year of full service. Testing of bus pad and guiding systems have not taken place yet. 


\section{Conclusion and discussion}

The project highlights some of the challenges that are often met when implanting universal design and accessibility in real-life physical projects.

Often a project is initiated with the highest ambitions - a new high-class bus system in one of the richest and most developed countries in the world should be $100 \%$ accessible to all user groups. And yet, a number of challenges were to be dealt with.

In the following, some of these challenges are described and discussed.

\subsection{Disparities in the guideline systems controlling the design of the stations and the buses}

In this case, both the designer of the platforms and the manufacturer of the buses followed valid guidelines and recognized best practice. The national Norwegian guidelines for road design prescribe a kerb height of $18 \mathrm{~cm}$, whereas the international guidelines on bus design allows a floor height - kneeling included - of $25-27 \mathrm{~cm}$, leaving a vertical gap that can't be negotiated by most wheelchair users. This means that both platform designers and bus manufacturers, understandably, were surprised to encounter the resulting real-life practical problems.

In a Danish context, the official guidelines dealing with different subjects, such as indoor as opposed to outdoor conditions, may give conflicting advice on the same subjects. And the national guidelines are not always aligned with international guidelines such as DIN/ISO standards.

The lack of consistency in the guidelines makes it difficult for non-experts to make the optimal choices and may - worst-case - discourage implementing universal design solutions.

Harmonization of guidelines could be an issue for the relevant authorities on both national and international level to consider in the future.

\subsection{Ambition and knowledge gap for implementing universal design}

The problem with the conflicting guidelines aside, there is often not sufficient expertise and knowledge available to the professionals directly in charge of implementing a project like the Metrobuss project.

In this project this fact is evidenced by the suggestion made during the process, that the manual ramp was considered sufficient to solve the problem. As described previously, this is perhaps a possible technical solution, but not a solution that meets the high demands set by the concept of universal design, as it does not facilitate unaided travel.

To build knowledge and awareness further training, information and communication, not only on the technical aspects of accessibility, but also on the more fundamental concepts of universal design and its underlying concept of providing a physical environment - including accessible means of transportation - that allows as many people as possible to lead and live a dignified and valuable life, is recommended. 


\subsection{Challenge: Ambitions on sustainable public transport clash with ambitions an accessibility.}

This project highlights a further challenge, that will probably need to be addressed many times going forward: It is generally recognized that making people change their mode of transport from individual cars to public transport is one of the tools needed to achieve a more sustainable society (ultimately fighting climate change etc).

Travel time and regularity are key parameters for people's choice of transport mode. A project like the Metrobuss system focuses exactly on these parameters. Travel speed is increased by building bus lanes and traffic signals that prioritize the buses. But achieving shorter stays at the bus stops is also part of this effort. Means to this can be abolishing ticket sale in the bus and allowing the passengers to enter and exit the bus through all doors.

Achieving access to the bus for a single wheelchair user e.g. by having the driver leave his place and using time to flip out a manual ramp will perhaps delay the bus a couple of minutes. This may not seem like much but may ultimately result in other passengers missing connections and reducing regularity in the whole system.

Motorized ramps take shorter time to deploy but may still cause delays.

The ideal solution in this case is probably a solution with a gap filler or short motorized ramp that is deployed every time the bus stops, and not only when a wheelchair user needs it. This would probably even shorten the average time at every bus stop, because passengers of all abilities would be able to enter or exit the bus faster. (In this case: A truly universal design where a technical solution aimed at helping people with disabilities actually help a much larger group - essentially everybody!)

\subsection{Conclusion}

The paper gives a short introduction to a recent process of implementing a high-class infrastructure project with high ambitions and some encountered challenges.

Everybody - expert and non-experts - can agree on the principle that public transport systems should be accessible on an equal basis to all users. With the ongoing fight against climate change, public transport is and will be a key component in nudging people to make more sustainable choices. And people with disabilities should not be left behind but - on the contrary - be able to take advantage of improved public transport systems.

The project from Trondheim shows the challenges that can arise when - among other factors - guidelines are not aligned, and the people involved are lacking knowledge about the ideas and principles of universal design. Through user and expert involvement various solutions can be found. And valuable lessons can be learned that may be used by other similar projects.

\section{References}

[1] The BRT standard, 2014 Edition

[2] Statens Vegvesen: Universell utformning av veger og gater, håndbok V129, 2011

[3] EU Bus directive 2001/85

[4] UN: Convention on the Rights of Persons with Disabilities (CRPD), article 2

[5] E-mail from Skånetrafiken, July $26^{\text {th }} .2019$

[6] Evalueringsrapport “Test af de elektriske ramper”, Fynbus, July 2019 